\title{
Genomic and phylogenetic analysis of Salmonella Typhimurium and its monophasic variants responsible for invasive endemic infections in Colombia
}

\author{
Yan Li ${ }^{1}$, Caisey V. Pulford ${ }^{1}$, Paula Díaz ${ }^{2}$, Blanca M. Perez-Sepulveda ${ }^{1}$, Carolina \\ Duarte $^{2}$, Alexander V. Predeus ${ }^{1}$, Magdalena Wiesner ${ }^{2}$, Darren Heavens ${ }^{3}$, Ross Low ${ }^{3}$, \\ Christian Schudoma ${ }^{3}$, James Lipscombe ${ }^{3}$, Angeline Montaño², Neil Hall ${ }^{3,4}$, Jaime \\ Moreno $^{2}$, Jay C. D. Hinton ${ }^{1^{*}}$. \\ 1 Institute of Integrative Biology, University of Liverpool, Liverpool, United Kingdom, \\ 2 Grupo de Microbiología, Instituto Nacional de Salud, Bogotá, Colombia \\ 3 Earlham Institute, Norwich, United Kingdom \\ 4 School of Biological Sciences, University of East Anglia, Norwich, UK \\ * jay.hinton@liverpool.ac.uk
}

\section{Abstract}

Salmonellosis is an endemic human infection, associated with both sporadic cases and outbreaks throughout Colombia. Typhimurium is the most common Colombian serovar of Salmonella enterica, responsible for $32.5 \%$ of the Salmonella infections. Whole genome sequencing (WGS) is being used increasingly in Europe and the USA to study the epidemiology of Salmonella, but there has not yet been a WGS-based analysis of Salmonella associated with bloodstream infection in Colombia. Here, we analysed 209 genome sequences of Colombian $S$. Typhimurium and monophasic $S .4,[5], 12: i$ :- isolates from Colombia from 1999 to 2017. We used a core genome-based maximum likelihood tree to define seven distinct clusters which were predominantly Sequence Type (ST) 19 isolates. We also identified the first ST313 and monophasic ST34 isolates to be reported in Colombia. The history of each cluster was reconstructed with a Bayesian tree to reveal a timeline of evolution. Cluster 7 was closely related to European multidrug-resistant (MDR) DT104. Cluster 4 became the dominant variant of Salmonella in 2016, and resistance to nalidixic acid was associated with a plasmid-encoded qnrB19 gene. Our findings suggest multiple transfers of $S$. Typhimurium between Europe and Colombia. 


\section{Author summary}

The large-scale genome sequencing of Salmonella Typhimurium and monophasic Salmonella 4,[5],12:i:- involved bloodstream isolates from Colombia. The two serovars were responsible for about $1 / 3$ of Salmonella infections in Colombia in the past 20 years. To identify the population structure we used Whole Genome Sequencing, performed in silico sequence typing, obtained phylogenetic trees, inferred the evolutionary history, detected the plasmids and prophages, and associated the antibiotic resistance (AMR) genotype with phenotype. Different clusters showed temporal replacement. The Colombian sequence type 313 was distinct from African lineages due to the absence of a key virulence-related gene, $b s t A$. One of the Colombian clusters is likely to belong to the global epidemic of DT104, according to the evolutionary history and the AMR profile. The most common cluster in recent years was resistant to nalidixic acid and carried a plasmid-mediated antibiotic resistant gene qnrB19. Our findings will inform the ongoing efforts to combat Salmonellosis by Colombian public health departments.

\section{Introduction}

Salmonella is a major food or water-borne pathogen that causes typhoid fever, invasive non-typhoidal disease or self-limiting gastroenteritis throughout the world. Salmonella enterica is divided into more than 2,600 serovars depending on the types of lipopolysaccharide ( $\mathrm{O}$ antigen) and flagellar protein ( $\mathrm{H}$ antigen) that are expressed the cell surface [1]. S. enterica serovar Typhimurium (Salmonella Typhimurium) is a major cause of non-typhoidal salmonellosis worldwide.Laboratory-based surveillanceby the Instituto Nacional de Salud (INS) of Colombia involved 12,055 S. enterica isolates from 1997 to 2017 , and found that $S$. Typhimurium was responsible for $28.4 \%$ of the cases identified by passive laboratory surveillance in the country. The majority $(54.9 \%)$ of the 1,302 Typhimurium isolates were multidrug resistant (MDR), and $32.4 \%$ were resistant to one or two antimicrobial agents. [2]

In recent years, whole genome sequencing has provided new insights to the epidemiology of Salmonella, especially in the UK [3] and the USA [4]. Almost 200,000 Salmonella genomes have been assembled and stored in Enterobase 
(https://enterobase.warwick.ac.uk/). The availability of thousands of whole-genome sequences of $S$. Typhimurium has given epidemiological investigators an unprecedented ability to discover outbreaks, which involve $S$. Typhimurium isolates that vary by fewer than five core-genome SNPs [5]. However, Enterobase only contained four genome sequences of Colombian $S$. Typhimurium.

Since the 1990s, monophasic variants of $S$. Typhimurium, S. 4,[5],12:i:- (MVST) have arisen at high frequency across the world. MVST is a variant of $S$. Typhimurium that does not express the phase $2 \mathrm{H}$-antigen [6]. The prevalence of MVST increased rapidly in Europe during the 2000s [7]. Two variants of MVST have been reported, suggesting convergent evolution: an ST19 "Spanish clone" and an ST34 "European clone" [8].

Recently, a new type of invasive non-typhoidal Salmonella (iNTS) causing highly fatal bloodstream infection has emerged in sub-Saharan Africa (SSA). iNTS disease is caused by two distinct lineages of $S$. Typhimurium ST313, both carrying MDR-encoding Tn21 elements on plasmid pSLT [9] and the prophages, BTP1 and BTP5 [10]. The acquisition of the cat chloramphenicol resistance gene by lineage 2 is thought to have played an important role in the clonal replacement of lineage 1, which occurred in Malawi and elsewhere in 2003 [9]. S. Typhimurium ST313 was recently found to cause $2.7 \%$ of the $S$. Typhimurium-associated gastroenteritis in the UK. A key feature that distinguishes UK-ST313 isolates from SSA ST313 lineage 2 is the absence of the BTP1 and BTP5 prophages [11]. It was not known if $S$. Typhimurium ST313 is also found in Colombia.

In the present study, we performed genomic and phylogenetic analysis of the whole genome sequences of 209 S. Typhimurium and MVST isolates from Colombia, 208 of which were sampled from patients with invasive blood infection. This bioinformatic dissection of the 209 genomes involved the analysis of core genome SNPS, AMR genes, plasmids and prophage profiles. We used a combination of maximum likelihood phylogenetic methods and bayesian inference to investigate the evolutionary history of $S$. Typhimurium and MVST in Colombia. 


\section{Materials and methods \\ Bacterial isolation and characterization}

All Salmonella isolates were collected and characterised at the Instituto Nacional de Salud (INS), Colombia. Two hundred and nine Salmonella isolates were selected for this study, including 208 isolates from human blood and one isolate from Hydrochoerus hydrochaeris, a South America-specific rodent. The isolates were obtained between 1999 and 2017 from 22 out of 33 Colombian departments. The serotyping was performed by INS according to the White-Kaufmann-Le Minor scheme [1], and identified $193 \mathrm{~S}$. Typhimurium and 16 S. 4,[5],12:i:- isolates.

Antimicrobial resistance (AMR) was determined using the Kirby-Bauer test and the Minimum Inhibitory Concentration (MIC) test by semi-automated MicroScan and Vitek 2 platforms, following the performance standards of the US Clinical and Laboratory Standards Institute [12]. The antimicrobials tested were ampicillin, chloramphenicol, streptomycin, tetracycline, gentamicin, amikacin, nalidixic acid, trimethoprim, ciprofloxacin, ceftazidime, and cefotaxime. The metadata of all isolates is summarised in S1 Table, including serovars, collection date, location, isolation source, and phenotypic AMR profile.

\section{Whole genome sequencing and assembly}

DNA extraction and whole genome sequencing was carried out at the Earlham Institute, Norwich, UK. DNA was extracted from $100 \mu \mathrm{l}$ of heat-killed bacterial culture using the MagAttract kit (Qiagen). Sequencing libraries were constructed using 1 ng of input DNA with a modified Nextera kit (Illumina). PCR amplified libraries were normalised and size-selected prior to sequencing on an Illumina HiSeq4000, with a 2x150 bp read metric and a median 30x genome coverage.

The adapter sequences of Illumina raw reads were trimmed using Trimmomatic v0.36 [13] in palindrome mode (ILLUMINACLIP:2:30:10). Quality trimming was conducted by Seqtk v1.2-r94 [14] using Phred algorithm. The genome assembly was performed with 
Unicycler v0.4.4 [15] with paired end and unpaired reads. The quality of assemblies was assessed by reference based Quast v4.6.3 [16]. A phage and plasmid-free Salmonella Typhimurium 4/74 genome (GenBank ID: CP002487) was used as Quast reference, in order to exclude the impact of variable regions of genome on quality assessment. N50 value and number of contigs were evaluated. The N50 value of all assemblies were $>15 \mathrm{~kb}$, and number of contigs were $<600$..

\section{Genome Analysis}

ARIBA v2.12.0 [17] and MLST v2.10 [18] were used for in silico sequence typing from raw reads and assembled data. Both analysis used PubMLST database [19] and based on the multilocus sequence typing (MLST) scheme of $S$. enterica, which defined the Salmonella sequence type on the basis of 7 housekeeping genes [20].

The assembled genomes were screened for AMR genes, plasmids, prophages, and flagellar genes. AMR genes and plasmids were identified using Abricate v0.8 [21] with Resfinder (https://cge.cbs.dtu.dk/services/ResFinder/) [22]and PlasmidFinder [23] database (coverage $>70 \%$ ). Prophages were searched using the online tool Phaster (http://phaster.ca) [24, 25]. Flagellar genes (UniProtKB identifiers: fljA: A0A0F7JBE2; fljB: Q549S3) were classified with Diamond v0.9.22 [26] using Blastx algorithm.

Genome annotation was performed using Prokka v1.13 [27]. Genome annotation data were passed to pan-genome analysis program Roary v3.12.0 [28] to build the core genome alignment and to define gene presence and absence. The core genome alignment was used for phylogenetic inference.

\section{Phylogenetic Analysis}

Single nucleotide polymorphisms (SNP) were identified with SNP-sites v2.4.0 [29], using the core gene alignment produced by Roary. To contextualize the Colombian $S$. Typhimurium isolates, 11 pre-existing genomes (Table 1) were downloaded from NCBI or Enterobase, and included in the core genome alignment. A maximum likelihood 
phylogenetic tree was inferred with Raxml-ng v0.6.0 [30] using the GTR+G model from the core genome SNP alignment with 100 bootstrap iterations. The tree was rooted using S. Typhi as an outgroup (GenBank accession number: AL513382.1). Lineage designations were assigned using population clustering by rhierBAPS v1.0.1 [31, 32]. The core genome SNP maximum likelihood tree was visualised with iTOL [33].

To understand the phylodynamics of the Colombian $S$. Typhimurium and MVST, BEAST v2.5.0 [40] was used to produce a Bayesian tree based on core gene alignment of individual clusters. Assuming the population size of $S$. Typhimurium and MVST in Colombia was constant across years, "constant population" was chosen as the coalescent tree prior. Different molecular clock models were compared to find the one that best fitted the data. Using Nested Sampling package v1.0.0 of BEAST [41], relaxed exponential clock model was supported by the marginal likelihood value. The BEAST analysis was run for 80 million steps and was sampled every 200 steps using the GTR substitution model with $\mathrm{y}$ correction for site specific variation. The initial 8 million steps were removed as burn-in. The target tree was summarized from the samples with TreeAnnotator and visualised with Figtree v1.4.3 [40].

\section{Data availability}

Fastq files are available at NCBI short Read Archive under the accession numbers listed in S1 table (BioProject Number PRJNA527650, SRA Study Number SRP188631.)

\section{Results and Discussion}

\section{Phylogenetic Analysis}

To determine the diversity of $S$. Typhimurium and MVST in Colombia, we performed in silico sequence typing in two ways, using ARIBA from raw reads and using MLST from assemblies. By combining both results (S1 Table), we identified six sequence types, including ST19 $(n=182)$, ST34 $(n=4)$, ST313 $(n=3)$, ST2936 $(n=1)$, ST2072 $(n=1)$ and ST1649 $(n=1)$. To our knowledge, this is the first report of ST313 in Colombia. ST313 is a major cause of invasive non-Typhoidal salmonellosis in Africa. ST34 was previously describe in two Colombian Typhimurium isolates harbouring mcr-1 gene, however, 
identification of this ST34 in blood samples, evidence a mayor spread in the country [42] and was a complement of the global finding of this monophasic sequence type in recent years [43-46]. The sequence type of 17 isolates were unable to be predicted from either method, due to incomplete sequencing of one of the seven MLST housekeeping genes. The low ST diversity found in these isolates are in agreement with $S$. Typhimurium taxonomy in which the vast majority of isolate are ST19, knowing as the ancestral type, in which most of the strains are associated with human gastroenteritis [47].

To determine the genetic structure of $S$. Typhimurium and MVST in Colombia we performed phylogenetic analysis of 220 genome sequences using maximum likelihood methods (Fig 1, S1 Fig). The analysis included 209 Colombian S. Typhimurium and MVST sequences and 11 contextual reference genomes, from which we identified 35,912 SNPs at the core genome level. Seven distinct clusters were identified using rHierBAPS and confirmed using phylogenetic clustering.

Cluster 1 comprised a monophasic ST34 clade, which included the DT120 and DT193 genomes as contextual. The contextual genomes were sampled from UK and defined as the "European clone" MVST with ASSuT resistance pattern (ampicillin, streptomycin, sulfonamide, and tetracycline) [39]. The majority of isolates in cluster 1 (16/17) were resistant to at least one antibiotic.

Cluster 2 was sampled from 1999 to 2017. The most common sequence type of cluster 2 was ST19, as well as a ST2072 and a ST1649 isolate. One cluster 2 isolate was very close to the contextual SALIA4985AA, which was collected in the USA from human stool.

All cluster 3 isolates were S. Typhimurium ST19, with the exception of three isolates which had unknown sequence type. All cluster 3 isolates showed resistance to at least one antibiotic in the AMR test.

Mixing of S. Typhimurium and MVST isolates was observed in cluster 4, indicating that the monophasic ST19 was caused by independent deletions of the $f / j A / B$ genes. All the monophasic ST19 isolates that belonged to cluster 4 were resistant to one or more antibiotics. 
Cluster 5 contained three ST313 and one ST2936 isolates, isolated between 2013 and 2017. ST2936 is a single locus variant of ST313 that differs by one MLST allele. All the ST313 isolates were sampled from infants under 2 years old or elderly people aged over 60 years old. The age characteristic of patients is accordant with the former report about the invasive non-typhoidal salmonellosis caused by Salmonella ST313, that the disease was associated with immunocompromised infants or senior patient [48].

Cluster 6 contained the isolate from Hydrochoerus hydrochaeris, CFS290-SF. 75.5\% of cluster 7 isolates were sampled in 2003-2007, and 79.2\% showed resistance to 3 or more antibiotics. Cluster 7 was closely-related to a representative DT104 isolate, with the minimal difference of only 22 core genome SNPs. The contextual DT104 isolate was sampled in Scotland, associated with MDR and wide-spread zoonotic infection [35].

The temporal variations between clusters are summarised in Fig 2a. It is clear that clusters 2, 6, and 7 were detected before 2002, whereas clusters 1 and 3 emerged in 2007, and clusters 4 and 5 appeared after 2011. Clusters 3 and 7 peaked during the years 2012-2014 and 2004-2007, respectively. The detection of cluster 4 rapidly increased in 2016. As the latest sampling period ended in 2017, it is possible that the recently emerged monophasic MDR ST19 could be an increasing threat to the health of the Colombian population.

The evolutionary history of Colombian S. Typhimurium was reconstructed by BEAST analysis. BEAST uses molecular clock models to estimate rooted, time-measured phylogenies. The topology of the Bayesian tree reconstructed from the Colombian $S$. Typhimurium genomes was consistent with the Raxml tree. The median value of the most recent common ancestor (MRCA) date of each cluster is shown in Fig $\mathbf{2 b}$.

The cluster 7 epidemic in Colombia in the early 2000s shared a high similarity with the contextual DT104 genome sampled from Scotland [35]. Specifically, the difference between DT104 and its closest relative in Colombia was only 25 core genome SNPs, while the branch length of cluster 7 was $>213$ SNPs. The Bayesian phylogenetic analysis of the Scottish DT104 predicted that the MRCA was dated to 1968 (95\% Highest posterior density (HPD): 1962 to 1976), while the prediction of the MRCA of cluster 7 was 1981 
(95\% HPD: 1956 to 1994), suggesting that the cluster 7 could be sourced from the Scottish DT104. We conclude that the multidrug resistant cluster 7 of Colombia was a part of the global epidemic of DT104 that began in the early 1980s, and appeared at a similar time in Europe, Japan, North American and Argentina[49].

\section{Accessory Genome}

As shown in Fig 3, a homology search with Blastx showed that $f l j A / B$ genes were absent from all MVST isolates. FljA is the phase-1 flagellin repressor, and FljB is phase2 flagellin. The deletion of either fljA/B gene will prevent the expression of phase $2 \mathrm{H}$ antigen, cause the generation of MVST [50].

Plasmids control important physiological functions of Salmonella, such as resistance to antibiotics and heavy metals, utilisation of carbon sources, and virulence factors [51]. As shown in Fig 3, incompatibility groups IncFIB(S) and IncFII(S) belongs to Salmonella Typhimurium Virulence Plasmid (pSTV),[52] were distributed in most of the Colombian Salmonella genomes, suggesting and association with blood-source isolates.

Although pSTV have been considered important for bacteremia cases in humans, their role in infection is still controversial. Heithoff and colleagues describe that Typhimurium isolates derived from human bacteremia harbored pSTV in contrast to isolates from gastroenteritis patients. However, several studies pointing that pSTV is not necessary for systemic infection [53]. In addition, an association between pSTV and ST19 over other STs was observed previously in Mexico Typhimurium strains, suggest that the presence of pSTV may be associated to different genetic traits [54]. The role of pSTV in bacteraemia and severe gastroenteritis remain scarce, however extensive studies in Typhimurium population will help to unravelling their involve in pathogenesis.

The other plasmids were usually concentrated in particular clusters. For example, small plasmids Col(MP18) and ColpVC were only in cluster 7, and IncQ and IncX were mainly possessed by cluster 1 and 3 . 
Prophages Gifsy-1 and Gifsy-2 are found in all major S. Typhimurium lineages [55] and were carried by most of the Colombian S. Typhimurium isolates (Fig. 3). Other prophages were carried by one or two clusters. For example, prophage P22 was mostly in cluster 4 and ST104 was only in cluster 7 . It is worth notice that Phaster only detected ST64T in ST313 and ST2936 isolates. The ST64T prophage shared a 22kb identical segment with BTP1 prophage carried by invasive African Typhimurium ST313 [10].

Fig 4 shows a comparison of a region of a Colombian ST313 genome with BTP1 and a BTP1-like prophage found in a UK ST313 strain [56]. The bstA gene (previously termed st313-td [57]) and the genes encoding the GtrA/C LPS-modifying enzymes were present in African BTP1, but absent from the Colombian BTP1-like prophage. GtrA/C is a glycosyltransferase enzyme that modifies the O-antigen to make invasive Salmonella resistant to BTP1 infection [58]. The comparison of BTP1 and BTP1-like prophages indicated that the Colombian ST313 was more similar to UK-ST313 than invasive African lineages. Further analysis of $S$. Typhimurium ST313 genomes across the world are likely to shed more light upon the evolutionary development of the life-threatening African Typhimurium ST313.

\section{AMR genotype and phenotype}

The result of antimicrobial susceptibility tests involving a panel of 11 drugs is summarised in S2 Table. The test showed that only $32.1 \%$ of the isolates were pansusceptible to all antimicrobials tested, while $35.8 \%$ were phenotypically resistant to one or two antimicrobials. $32.1 \%$ were resistant to three or more antimicrobials. Tetracycline resistance was the most common resistance phenotype, found in more than a half of the isolates $(54.6 \%, n=114)$, followed by streptomycin resistance $(36.4 \%, n=76)$, ampicillin resistance $(29.2 \%, n=61)$ and chloramphenicol resistance $(29.2 \%, n=61)$. Fig 5 compared the four most common antibiotic resistance in the Colombian $S$. Typhimurium and MVST isolates.

Fig 6 shows the comparison of antimicrobial resistant phenotypes and genotypes of the 7 clusters of Colombian S. Typhimurium and MVST. Tetracycline resistance was seen 
in all the seven clusters, and was more concentrated in cluster 1,3,4,7. Except the streptomycin resistance isolates $(n=76)$, there were high proportion of isolates showed intermediate resistance to streptomycin $(n=58)$. Resistance to ampicillin, chloramphenicol and nalidixic acid was concentrated in one or two clusters.

The MDR phenotype of cluster 7 was associated with two AMR gene patterns: A clade resistance to ampicillin, chloramphenicol, streptomycin, and tetracycline was related to the presence of $\operatorname{aad} A 2$, blaCARB-2, floR, tet(A) and tet(G); and the other clade was resistant to ampicillin, tetracycline and trimethoprim carried aph(3')-la, blaOXA-2, dfrA29, mphB, sul1, and tet(A). The former clade shared the similar AMR phenotype and genotype as the MDR DT104 that emerged globally in the 1990s, which also carried the aadA, floR, sul1 and tet (G) genes [59].

Although the phylogenetic analysis showed that the Colombian MVST ST34 isolates were in the same clade as the representative monophasic ST34 "European clone" DT120 and DT193, there was obvious distinction in terms of AMR phenotypes and genotypes. Unlike the "European clone" with ASSUT resistance pattern, the Colombian ST34 did not show resistance to more than three drugs. Three of the four Colombian ST34 isolates were susceptible to ampicillin. Three of the isolates were resistant to tetracycline and carried the $\operatorname{tet}(B)$ gene.

The most recent cluster to have emerged was cluster 4. A plasmid mediated quinolone resistance gene, qnrB19 [60], was associated with cluster 4. In vitro antimicrobial susceptibility testing confirmed that the majority of the cluster 4 isolates were resistant to nalidixic acid and also showed intermediate resistance to ciprofloxacin. Both drugs have been listed as highest priority by the WHO in the context of combatting antimicrobial resistance [61].

The qnrB19 gene was encoded by a small ColRNAl plasmid. Four different qnrB19-harboring ColRNAI plasmids were recovered from the Unicycler assemblies of Colombian Salmonella genomes (S2 Fig). One was identical to pMK100 (GenBank ID: HM070379.1), a 2,699bp plasmid which had previously been identified in a Colombian isolate of Salmonella Infantis, sampled from retail chicken in 2004 [62]. 


\section{Conclusion}

Following the sequencing of 209 S. Typhimurium and MVST genomes from Colombian isolates from 1999-2017, building phylogenetic trees and inferring the evolutionary history, we identified 7 clusters of $S$. Typhimurium and MVST that were associated with bloodstream infection. We detected cluster-specific patterns of plasmids and prophages in Colombian S. Typhimurium and MVST. The AMR genes responsible for antibiotic resistance phenotypes were identified from the genome data.

We identified a clade in cluster 7 with a characteristic MDR profile of ampicillin, chloramphenicol, streptomycin, and tetracycline. The clade shared high core genome similarity with a contextual DT104 genome from Scotland. From the identity of AMR genotype and phenotype and the estimated year of MRCA, we infer that the cluster 7 clade belonged to the global pandemic of MDR DT104, which spread across Europe, Asia and America from the 1970s [59].

Three Colombian S. Typhimurium ST313 isolates associated with bloodstream infection were sampled from infant and senior patients. Unlike the ST313 lineage 2 which is causing fatal invasive nontyphoidal salmonella diseases in Africa, the Colombian ST313 did not carry the African version of the BTP1 prophage. Our analysis suggests that the Colombian ST313 are more closely related to be related to the UK-ST313 lineages, which have been associated with gastroenteritis in England.

The emergence of MDR monophasic ST34 was widely reported in European, Asia, Australia, and North America in recent years [43-46]. However, the Colombian ST34 differed from the European MVST strains in terms of the antibiotic-resistant phenotype and genotype. None of the Colombian ST34 isolates showed resistance to more than 3 drugs. The monophasic ST19 isolates were distributed between clusters 2, 4 and 6, which suggests that the deletion of $f \mid j A / B$ genetic region could have occurred independently in Colombia, or reflects horizontal gene transfer.

Increasing numbers of cluster 4 isolates were detected in 2015 and 2016, with characteristic resistance to nalidixic acid and intermediate resistance to ciprofloxacin, linked to the plasmid-encoded quinolone resistance gene qnrB19. The potential spread 
of qnrB19 gene caused by the dominance of cluster 4 and the likelihood of transfer of CoIRNAI plasmids could be significant for Colombian public health in the future.

By analysing the relatedness of Colombian $S$. Typhimurium isolates from human bloodstream over an eighteen year period, we have identified a number of phylogenetic clusters that were also found in Europe. For the future, it will be interesting to learn more about the factors involved in the interchange of $S$. Typhimurium between Europe and Latin America.

\section{Supporting information}

S1 Table.

The metadata of all the samples used.

S2 Table.

Phenotypic determination of AMR profiles.

S1 Fig.

The detailed Raxml tree (including bootstrap values) and the BEAST tree.

S2 Fig.

The small plasmids that carry the qnrB quinolone-resistance gene. The plasmid 1-4 was possessed by isolate 458, GMR-S-816, GMR-S-1160, and 375 separately.

\section{Acknowledgements}

This work was supported by a BBSRC/GCRF award to $\mathrm{NH}$ and JCDH (BBS/OS/GC/000009D) and by a Wellcome Trust Senior Investigator award to JCDH (Grant 106914/Z/15/Z). NH is supported by the BBSRC Core Strategic Programme Grant BB/CSP17270/1. DH and $\mathrm{JL}$ are supported by the Earlham National Capability in Genomics (BB/CCG1720/1). 


\section{References}

1. Grimont PAD, Weill F-X. Antigenic formulae of the Salmonella serovars. WHO collaborating centre for reference and research on Salmonella. 2007;9:1-166.

2. Salud INd. Vigilancia Por Laboratorio De Salmonella spp. 20172018.

3. Ashton PM, Nair S, Peters TM, Bale JA, Powell DG, Painset A, et al. Identification of Salmonella for public health surveillance using whole genome sequencing. PeerJ. 2016;4:e1752. doi: 10.7717/peerj.1752.

4. Henk CdB, Marc WA, Dianna B, Eric WB, John F, Zamin I, et al. Rapid Whole-Genome Sequencing for Surveillance of $<$ em $>$ Salmonella enterica $</$ em $>$ Serovar Enteritidis. Emerging Infectious Disease journal. 2014;20(8):1306. doi: 10.3201/eid2008.131399.

5. Painset A, Jenkins C, Hartman H, Grant K, Dallman T, Ashton P, et al. SnapperDB: a database solution for routine sequencing analysis of bacterial isolates. Bioinformatics. 2018;34(17):3028-9. doi: 10.1093/bioinformatics/bty212.

6. Switt AIM, Soyer Y, Warnick LD, Wiedmann M. Emergence, Distribution, and Molecular and Phenotypic Characteristics of Salmonella enterica Serotype 4,5,12:i:-. Foodborne Pathogens and Disease. 2009;6:407-15. doi: 10.1089/fpd.2008.0213. 7. Hopkins KL, Kirchner M, Guerra B, Granier SA, Lucarelli C, Porrero MC, et al. Multiresistant Salmonella enterica serovar 4,[5],12:i:- in Europe: a new pandemic strain? Eurosurveillance. 2010;15. doi: https://doi.org/10.2807/ese.15.22.19580-en.

8. Arai N, Sekizuka T, Tamamura Y, Tanaka K, Barco L, Izumiya H, et al. Phylogenetic characterization of Salmonella enterica serovar Typhimurium and its monophasic variant isolated from food animals in Japan revealed replacement of major epidemic clones in the last four decades. Journal of clinical microbiology. 2018:JCM. 01758-17.

9. Okoro CK, Kingsley RA, Connor TR, Harris SR, Parry CM, Al-Mashhadani MN, et al. Intra-continental spread of human invasive Salmonella Typhimurium pathovariants in subSaharan Africa. Nature genetics. 2012;44:1215-21. doi: 10.1038/ng.2423.

10. Owen SV, Wenner N, Canals R, Makumi A, Hammarlöf DL, Gordon MA, et al. Characterization of the Prophage Repertoire of African Salmonella Typhimurium ST313 Reveals High Levels of Spontaneous Induction of Novel Phage BTP1. Frontiers in Microbiology. 2017;8:235. doi: 10.3389/fmicb.2017.00235.

11. Ashton PM, Owen SV, Kaindama L, Rowe WPM, Lane CR, Larkin L, et al. Public health surveillance in the UK revolutionises our understanding of the invasive Salmonella Typhimurium epidemic in Africa. Genome Medicine. 2017;9:92. doi: 10.1186/s13073-0170480-7.

12. Patel JB. Performance standards for antimicrobial susceptibility testing. 2017.

13. Bolger AM, Lohse M, Usadel B. Trimmomatic: a flexible trimmer for Illumina sequence data. Bioinformatics. 2014;30:2114-20. doi: 10.1093/bioinformatics/btu170.

14. Seqtk: Toolkit for processing sequences in FASTA/Q formats, (2013).

15. Wick RR, Judd LM, Gorrie CL, Holt KE. Unicycler: Resolving bacterial genome assemblies from short and long sequencing reads. PLOS Computational Biology. 2017;13:e1005595. doi: 10.1371/journal.pcbi.1005595.

16. Gurevich A, Saveliev V, Vyahhi N, Tesler G. QUAST: quality assessment tool for genome assemblies. Bioinformatics. 2013;29:1072-5. doi: 10.1093/bioinformatics/btt086. 
17. Hunt M, Mather AE, Sánchez-Busó L, Page AJ, Parkhill J, Keane JA, et al. ARIBA: rapid antimicrobial resistance genotyping directly from sequencing reads. Microbial Genomics. 2017;3:-.

18. MLST: Scan contig files against PubMLST typing schemes, (2018).

19. Jolley KA, Maiden MCJ. BIGSdb: Scalable analysis of bacterial genome variation at the population level. BMC Bioinformatics. 2010;11:595. doi: 10.1186/1471-2105-11-595.

20. Achtman M, Wain J, Weill F-X, Nair S, Zhou Z, Sangal V, et al. Multilocus Sequence Typing as a Replacement for Serotyping in Salmonella enterica. PLOS Pathogens. 2012;8:e1002776. doi: 10.1371/journal.ppat.1002776.

21. ABRicate: Mass screening of contigs for antimicrobial and virulence genes, (2018).

22. Aarestrup FM, Hasman H, Vestergaard M, Zankari E, Larsen MV, Lund O, et al. Identification of acquired antimicrobial resistance genes. Journal of Antimicrobial Chemotherapy. 2012;67(11):2640-4. doi: 10.1093/jac/dks261.

23. Carattoli A, Zankari E, García-Fernández A, Voldby Larsen M, Lund O, Villa L, et al. $<$ em $>$ In Silico $</$ em $>$ Detection and Typing of Plasmids using PlasmidFinder and Plasmid Multilocus Sequence Typing. Antimicrobial Agents and Chemotherapy. 2014;58(7):3895903. doi: 10.1128/aac.02412-14.

24. Zhou Y, Liang Y, Lynch KH, Dennis JJ, Wishart DS. PHAST: a fast phage search tool. Nucleic Acids Res. 2011;39:W347-52. doi: 10.1093/nar/gkr485.

25. Arndt D, Grant JR, Marcu A, Sajed T, Pon A, Liang Y, et al. PHASTER: a better, faster version of the PHAST phage search tool. Nucleic Acids Res. 2016;44:W16-21. doi: 10.1093/nar/gkw387.

26. Buchfink B, Xie C, Huson DH. Fast and sensitive protein alignment using DIAMOND. Nature Methods. 2014;12:59. doi: 10.1038/nmeth.3176.

27. Seemann T. Prokka: rapid prokaryotic genome annotation. Bioinformatics. 2014;30:2068-9. doi: 10.1093/bioinformatics/btu153.

28. Page AJ, Cummins CA, Hunt M, Wong VK, Reuter S, Holden MTG, et al. Roary: rapid large-scale prokaryote pan genome analysis. Bioinformatics. 2015;31:3691-3. doi: 10.1093/bioinformatics/btv421.

29. Page AJ, Taylor B, Delaney AJ, Soares J, Seemann T, Keane JA, et al. SNP-sites: rapid efficient extraction of SNPs from multi-FASTA alignments. Microbial Genomics. 2016;2:-. doi: 10.1099/mgen.0.000056.

30. Kozlov A, Darriba D, Flouri T, Morel B, Stamatakis A. RAxML-NG: A fast, scalable, and user-friendly tool for maximum likelihood phylogenetic inference. bioRxiv. 2018. doi: 10.1101/447110.

31. Tonkin-Hill G, Lees JA, Bentley SD, Frost SDW, Corander J. RhierBAPS: An R implementation of the population clustering algorithm hierBAPS [version 1; referees: 2 approved]. Wellcome Open Research. 2018;3. doi: 10.12688/wellcomeopenres.14694.1. 32. Cheng L, Connor TR, Sirén J, Aanensen DM, Corander J. Hierarchical and Spatially Explicit Clustering of DNA Sequences with BAPS Software. Molecular Biology and Evolution. 2013;30:1224-8. doi: 10.1093/molbev/mst028.

33. Letunic I, Bork P. Interactive tree of life (iTOL) v3: an online tool for the display and annotation of phylogenetic and other trees. Nucleic Acids Research. 2016;44:W242-W5. doi: 10.1093/nar/gkw290. 
34. Jarvik T, Smillie C, Groisman EA, Ochman H. Short-Term Signatures of Evolutionary Change in the Salmonella enterica Serovar Typhimurium 14028 Genome. Journal of Bacteriology. 2010;192:560-7. doi: 10.1128/JB.01233-09.

35. Mather AE, Reid SWJ, Maskell DJ, Parkhill J, Fookes MC, Harris SR, et al.

Distinguishable Epidemics of Multidrug-Resistant Salmonella Typhimurium DT104 in Different Hosts. Science. 2013;341:1514-7. doi: 10.1126/science.1240578.

36. McClelland M, Sanderson KE, Spieth J, Clifton SW, Latreille P, Courtney L, et al. Complete genome sequence of Salmonella enterica serovar Typhimurium LT2. Nature. 2001;413:852. doi: 10.1038/35101614.

37. Kröger C, Dillon SC, Cameron ADS, Papenfort K, Sivasankaran SK, Hokamp K, et al. The transcriptional landscape and small RNAs of Salmonella enterica serovar Typhimurium. Proceedings of the National Academy of Sciences of the United States of America. 2012;109:E1277-E86. doi: 10.1073/pnas.1201061109.

38. Luo Y, Kong Q, Yang J, Golden G, Wanda S-Y, Jensen RV, et al. Complete Genome Sequence of the Universal Killer Salmonella enterica Serovar Typhimurium UK-1 (ATCC 68169). Journal of Bacteriology. 2011;193:4035-6. doi: 10.1128/JB.05224-11.

39. Petrovska L, Mather AE, AbuOun M, Branchu P, Harris SR, Connor T, et al. Microevolution of monophasic Salmonella typhimurium during epidemic, United Kingdom, 2005-2010. Emerging infectious diseases. 2016;22:617.

40. Bouckaert R, Heled J, Kühnert D, Vaughan T, Wu C-H, Xie D, et al. BEAST 2: A Software Platform for Bayesian Evolutionary Analysis. PLOS Computational Biology. 2014;10:e1003537. doi: 10.1371/journal.pcbi.1003537.

41. Brewer BJ, Russel PM, Klaere S, Bouckaert RR. Model Selection and Parameter Inference in Phylogenetics Using Nested Sampling. Systematic Biology. 2018;68(2):219-33. doi: 10.1093/sysbio/syy050.

42. Saavedra SY, Diaz L, Wiesner M, Correa A, Arévalo SA, Reyes J, et al. Genomic and Molecular Characterization of Clinical Isolates of $<$ span class="named-content genusspecies" id="named-content-1" $>$ Enterobacteriaceae $</$ span $>$ Harboring $<$ em $>$ mcr $-1</$ em $>$ in Colombia, 2002 to 2016. Antimicrobial Agents and Chemotherapy. 2017;61(12):e0084117. doi: $10.1128 /$ aac.00841-17.

43. Alicia A, Qinning W, Nathan B, Rosemarie S, Chayanika B, Cristina S, et al. MultidrugResistant <em>Salmonella</em><em>enterica</em> 4,[5],12:i:- Sequence Type 34, New South Wales, Australia, 2016-2017. Emerging Infectious Disease journal. 2018;24(4):751. doi: 10.3201/eid2404.171619.

44. Mourão J, Machado J, Novais C, Antunes P, Peixe L. Characterization of the emerging clinically-relevant multidrug-resistant Salmonella enterica serotype 4,[5],12:i:-

(monophasic variant of S. Typhimurium) clones. European Journal of Clinical Microbiology \& Infectious Diseases. 2014;33(12):2249-57. doi: 10.1007/s10096-014-2180-1.

45. Tabor H, Gilmour M, McCracken M, Ahmed R, Langner S, Mulvey MR, et al. Emergence of multidrug-resistant Salmonella enterica serotype 4,[5],12:i:- involving human cases in Canada: results from the Canadian Integrated Program on Antimicrobial Resistance Surveillance (CIPARS), 2003-10. Journal of Antimicrobial Chemotherapy. 2013;68(9):1982-6. doi: 10.1093/jac/dkt149.

46. Mather AE, Phuong TLT, Gao Y, Clare S, Mukhopadhyay S, Goulding DA, et al. New Variant of Multidrug-Resistant \&lt;em\&gt;Salmonella enterica\&lt;/em\&gt; Serovar 
Typhimurium Associated with Invasive Disease in Immunocompromised Patients in Vietnam. mBio. 2018;9(5):e01056-18. doi: 10.1128/mBio.01056-18.

47. Branchu P, Bawn M, Kingsley RA. Genome Variation and Molecular Epidemiology of $<$ span class="named-content genus-species" id="named-content-1">Salmonella enterica</span> Serovar Typhimurium Pathovariants. Infection and Immunity. 2018;86(8):e00079-18. doi: 10.1128/iai.00079-18.

48. Feasey NA, Dougan G, Kingsley RA, Heyderman RS, Gordon MA. Invasive nontyphoidal salmonella disease: an emerging and neglected tropical disease in Africa. The Lancet. 2012;379(9835):2489-99. doi: https://doi.org/10.1016/S0140-6736(11)61752-2. 49. Helms M, Ethelberg S, Mølbak K, Group DS, others. International Salmonella typhimurium DT104 infections, 1992--2001. Emerging infectious diseases. 2005;11:859. 50. Mastrorilli E, Pietrucci D, Barco L, Ammendola S, Petrin S, Longo A, et al. A Comparative Genomic Analysis Provides Novel Insights Into the Ecological Success of the Monophasic Salmonella Serovar 4,[5],12:i. Frontiers in Microbiology. 2018;9:715. doi: 10.3389/fmicb.2018.00715.

51. Pilla G, Tang CM. Going around in circles: virulence plasmids in enteric pathogens. Nature Reviews Microbiology. 2018;16(8):484-95. doi: 10.1038/s41579-018-0031-2.

52. Feng Y, Liu J, Li Y-G, Cao F-L, Johnston RN, Zhou J, et al. Inheritance of the Salmonella virulence plasmids: Mostly vertical and rarely horizontal. Infection, Genetics and Evolution. 2012;12(5):1058-63. doi: https://doi.org/10.1016/i.meegid.2012.03.004.

53. Silva C, Puente JL, Calva E. Salmonella virulence plasmid: pathogenesis and ecology. Pathogens and Disease. 2017;75(6). doi: 10.1093/femspd/ftx070.

54. Wiesner M, Zaidi MB, Calva E, Fernández-Mora M, Calva JJ, Silva C. Association of virulence plasmid and antibiotic resistance determinants with chromosomal multilocus genotypes in Mexican Salmonella enterica serovar Typhimurium strains. BMC

Microbiology. 2009;9(1):131. doi: 10.1186/1471-2180-9-131.

55. Hiley L, Fang N-X, Micalizzi GR, Bates J. Distribution of Gifsy-3 and of Variants of ST64B and Gifsy-1 Prophages amongst Salmonella enterica Serovar Typhimurium Isolates: Evidence that Combinations of Prophages Promote Clonality. PLOS ONE.

2014;9(1):e86203. doi: 10.1371/journal.pone.0086203.

56. No Title, (2018).

57. Herrero-Fresno A, Wallrodt I, Leekitcharoenphon P, Olsen JE, Aarestrup FM, Hendriksen RS. The Role of the st313-td Gene in Virulence of Salmonella Typhimurium ST313. PLOS ONE. 2014;9(1):e84566. doi: 10.1371/journal.pone.0084566.

58. Kintz E, Davies MR, Hammarlöf DL, Canals R, Hinton JCD, van der Woude MW. A BTP1 prophage gene present in invasive non-typhoidal Salmonella determines composition and length of the 0 -antigen of the lipopolysaccharide. Molecular Microbiology. 2015;96:263-75. doi: 10.1111/mmi.12933.

59. Leekitcharoenphon P, Hendriksen RS, Le Hello S, Weill F-X, Baggesen DL, Jun S-R, et al. Global Genomic Epidemiology of Salmonella enterica Serovar Typhimurium DT104. Applied and Environmental Microbiology. 2016;82:2516-26. doi: 10.1128/AEM.03821-15. 60. Wang M, Jacoby GA, Mills DM, Hooper DC. SOS Regulation of qnrB Expression. Antimicrobial Agents and Chemotherapy. 2009;53:821-3. doi: 10.1128/AAC.00132-08. 61. Organization WH. Critically important antimicrobials for human medicine: ranking of antimicrobial agents for risk management of antimicrobial resistance due to non-human use2017. 
62. Karczmarczyk M, Leonard N, Martins M, McCusker M, Fanning S, Amaral L, et al. Characterization of antimicrobial resistance in Salmonella enterica food and animal isolates from Colombia: identification of a qnrB19-mediated quinolone resistance marker in two novel serovars. FEMS Microbiology Letters. 2010;313(1):10-9. doi: 10.1111/j.15746968.2010.02119.x. 
bioRxiv preprint doi: https://doi.org/10.1101/588608; this version posted March 25, 2019. The copyright holder for this preprint (which was not certified by peer review) is the author/funder, who has granted bioRxiv a license to display the preprint in perpetuity. It is made available under aCC-BY 4.0 International license.

Table 1: Contextual isolate details.

\begin{tabular}{|l|l|l|l|l|l|}
\hline Strain & $\begin{array}{l}\text { GenBank / } \\
\text { Enterobase ID }\end{array}$ & $\begin{array}{l}\text { NCBI Biosample } \\
\text { ID }\end{array}$ & $\begin{array}{l}\text { Sequence } \\
\text { Type }\end{array}$ & Country & Reference \\
\hline 14028s & GCA_000022165.1 & SAMN02604137 & ST19 & USA & {$[34]$} \\
\hline DT104 & GCA_000493675.1 & SAMEA2272504 & ST19 & UK & {$[35]$} \\
\hline LT2 & GCA_000006945.2 & SAMN02604315 & ST19 & USA & {$[36]$} \\
\hline SALIA4985AA & SALEA3995AA & SAMN03795441 & ST19 & USA & NA \\
\hline SALIA8156AA & SALCA0005AA & SAMN03479078 & ST19 & UK & NA \\
\hline SALJA4500AA & SALCA8991AA & SAMN02843993 & ST19 & Chile & NA \\
\hline SL1344 & GCA_000210855.2 & SAMEA3138382 & ST19 & UK & {$[37]$} \\
\hline UK1 & GCA_000213635.1 & SAMN02602986 & ST19 & USA & {$[38]$} \\
\hline U2 & GCA_900184385.1 & SAMEA104087369 & ST313 & UK & {$[11]$} \\
\hline DT120 & SAL_DA4862AA & SAMEA788645 & ST34 & UK & {$[39]$} \\
\hline DT193 & SAL_DA4867AA & SAMEA788680 & ST34 & UK & {$[39]$} \\
\hline
\end{tabular}



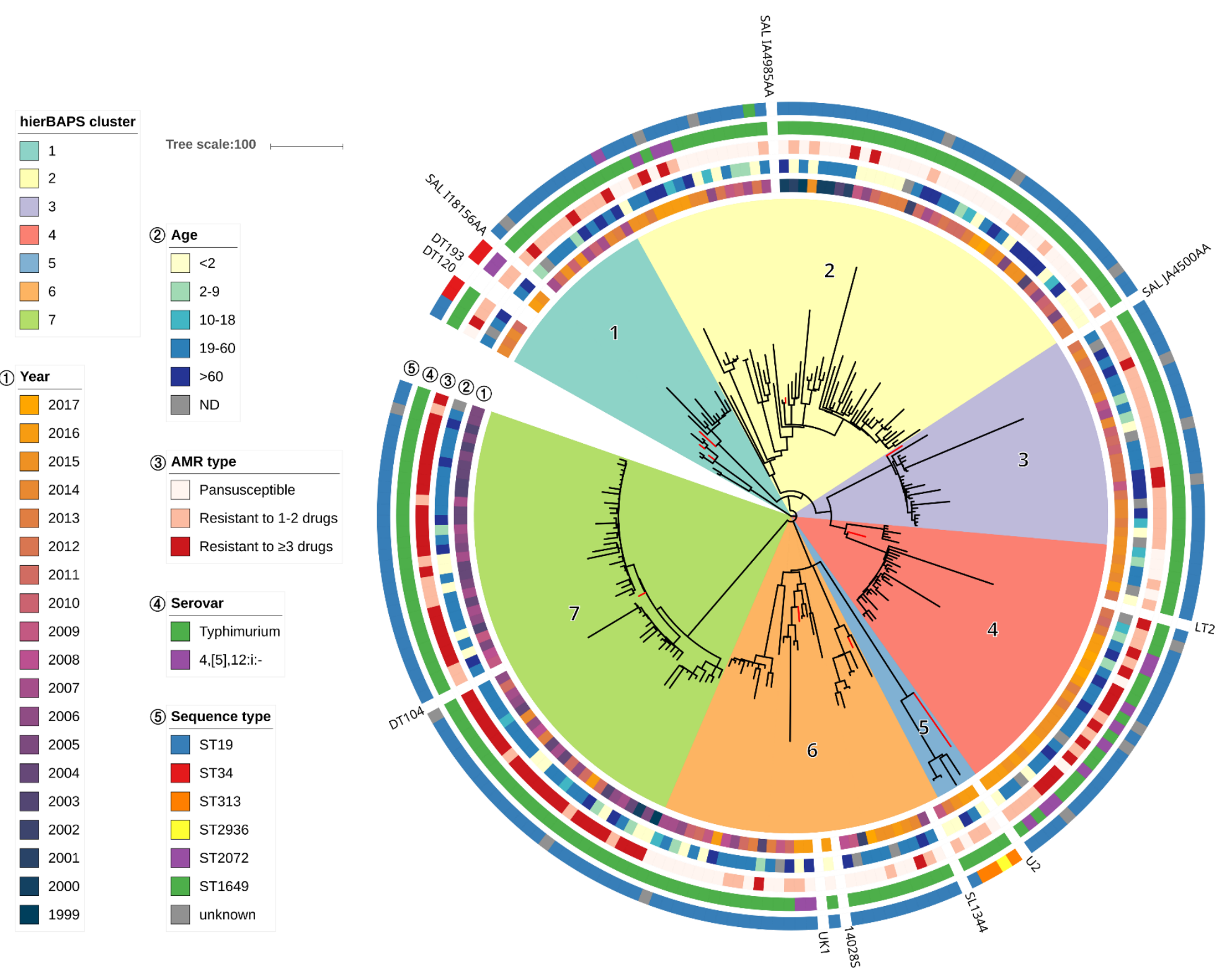

Fig 1. Maximum-likelihood phylogeny of 209 S. Typhimurium and MVST isolates from Colombia. 11 contextual genomes are shown as red branches. The hierBAPS-defined clusters 1-7, year of sampling, ages of patients, AMR types, serovars and sequence types are shown. The scale bar represents the number of SNPs per branch. 


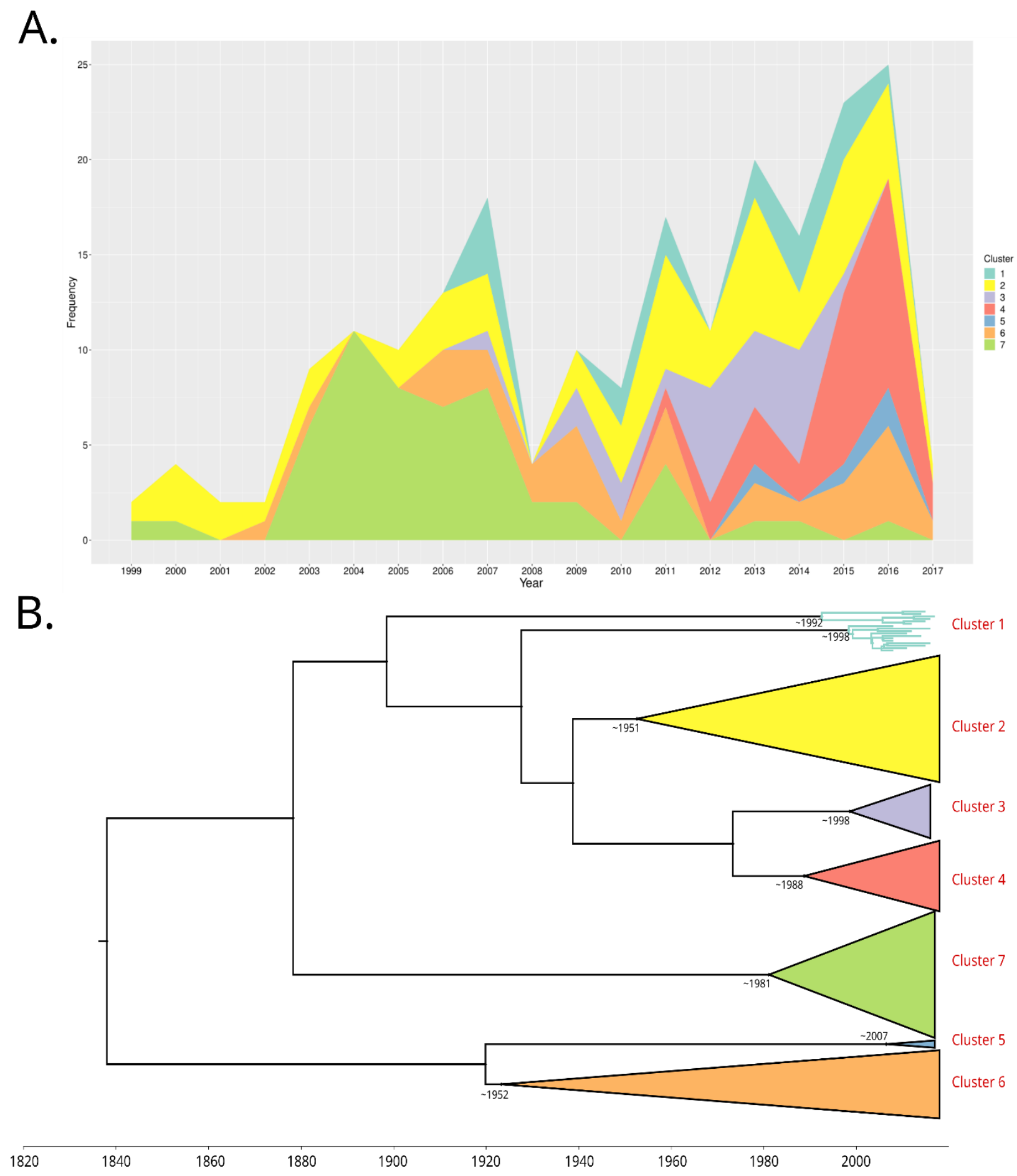

Fig 2. Temporal variations between clusters and the Bayesian phylogeny of 209 Colombia isolates. A. A line chart plotted with $R$ shows different clusters by colours. $X$ axis and $Y$ axis represent the sampling year and frequency. The latest sample was in 2017; B. Each cluster is collapsed in the Bayesian tree. Time scale was marked in decade. 
bioRxiv preprint doi: https://doi org/10.1101/588608; this version posted March 25, 2019. The copyright holder for this preprint (which was not

Tree scale:100

\section{Sequence type}

ST19

ST34

ST313

\section{ST2936}

ST2072

ST1649

unknown

\section{Serovar}

Typhimurium

"4,[5],12:i:-"

Fig 3. Distribution of flagella genes, plasmids, and prophages. Clusters 1-7, serovars and sequence types are indicated with colours. Red and grey show the presence and absence of flagella genes, plasmids, and prophages, respectively. 


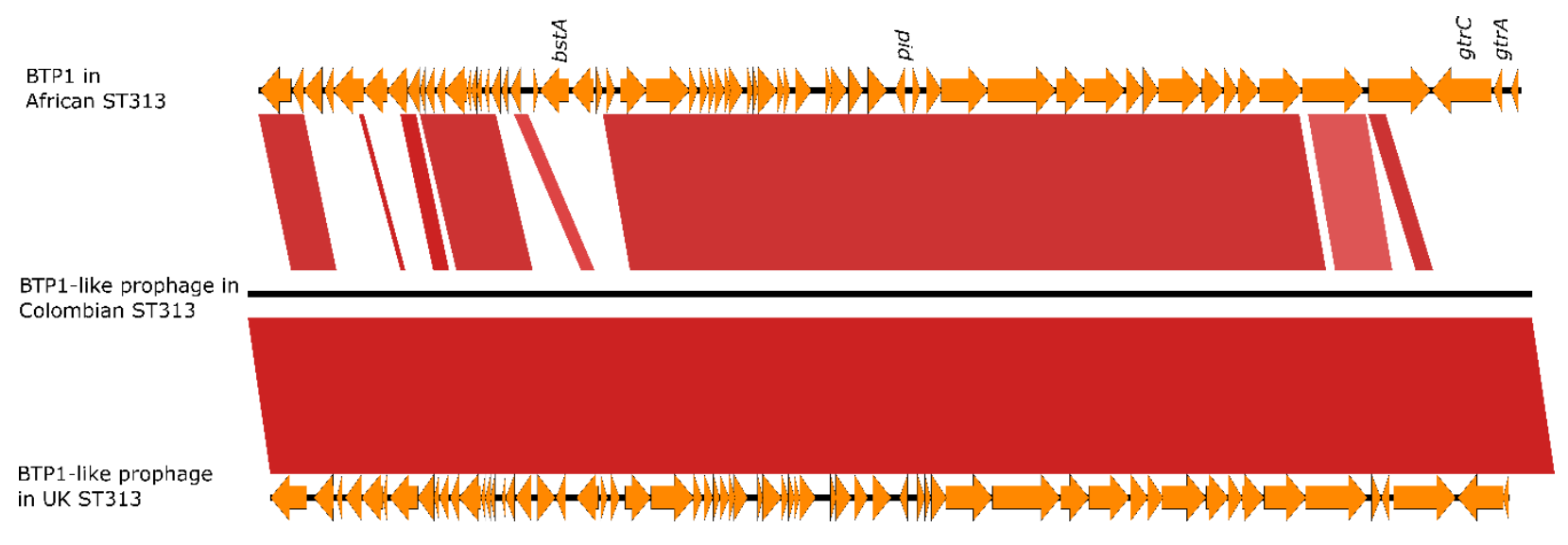

Fig 4. Multigenome comparison of BTP1/BTP1-like prophage region of African ST313 strain D23580, Colombia ST313 isolate 505, and UK ST313 isolate U15. Arrows represent coding sequences. Dark red blocks show regions with high genetic similarity. The position of $b s t A$, pid, gtrA/C genes are labelled on the BTP1 genome. 


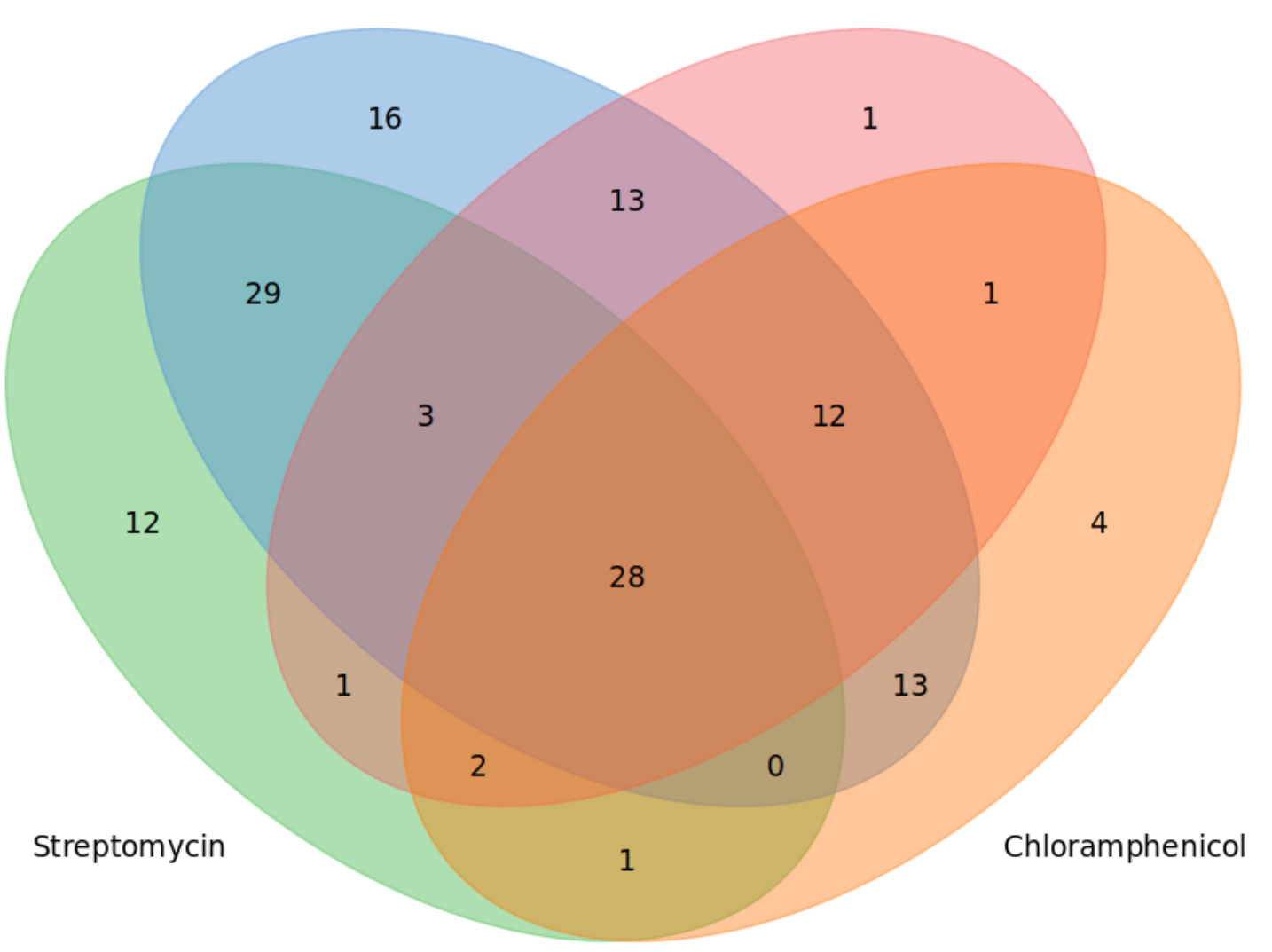

Fig 5. Venn diagram demonstrating the significant overlap of Salmonella isolates that resistant to ampicillin, chloramphenicol, tetracycline and streptomycin. 

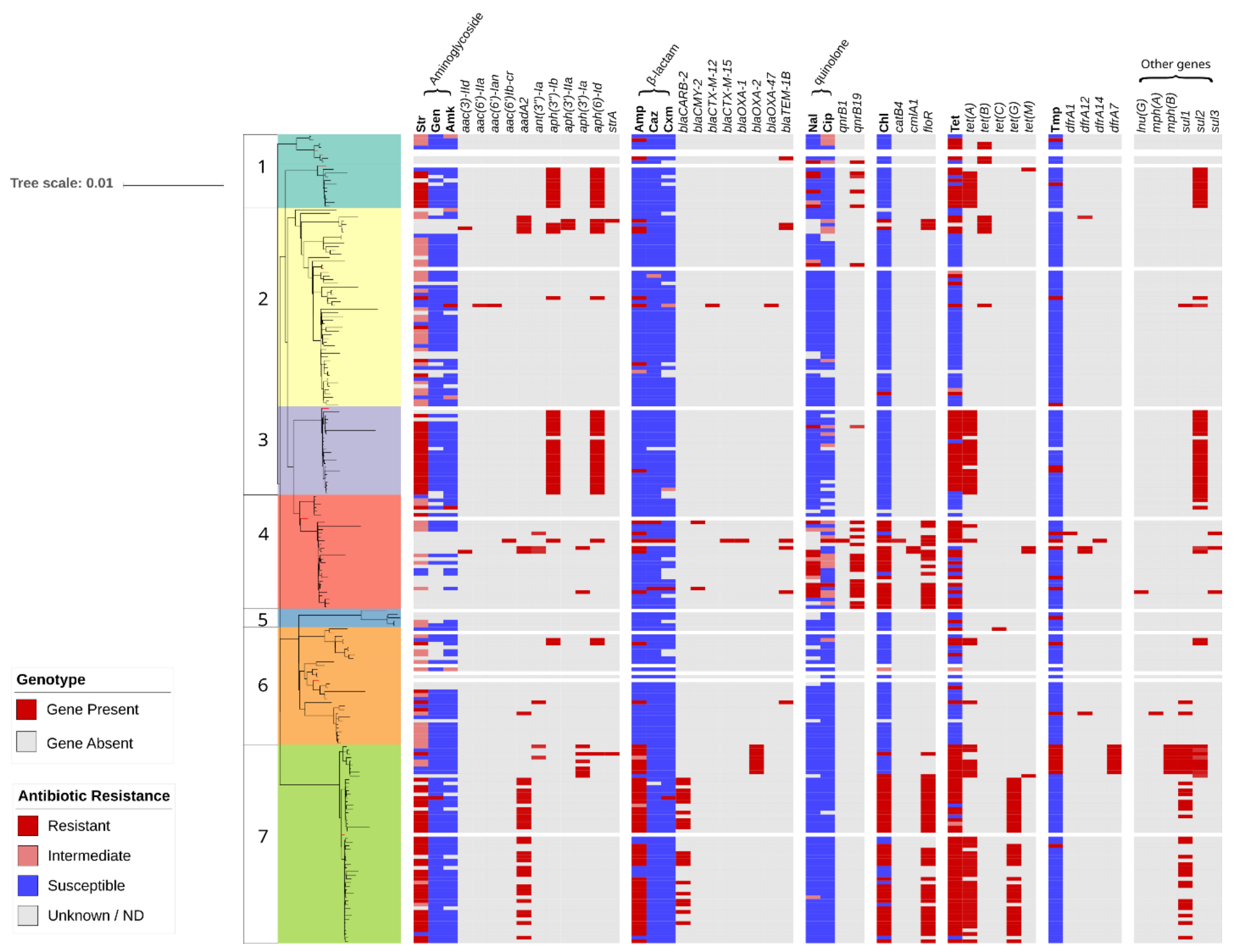

Fig 6. The comparison of antibiotic resistance phenotype and Genotype.

Clusters 1-7 were indicated with colours. The presence and absence of AMR genes were indicated in red and grey. Red, pink, blue and grey indicated the result of antibiotic resistance test. The abbreviation of antibiotics: amp: ampicillin, chl: chloramphenicol, str: streptomycin, tet: tetracycline, gen: gentamicin, amk: amikacin, nal: nalidixic acid, tmp: trimethoprim, cip: ciprofloxacin, caz: ceftazidime, cxm: cefotaxime. The 11 antibiotics are classified into 6 groups according to their chemical property. There are 6 genes related to antibiotics that did not be tested in this study. 